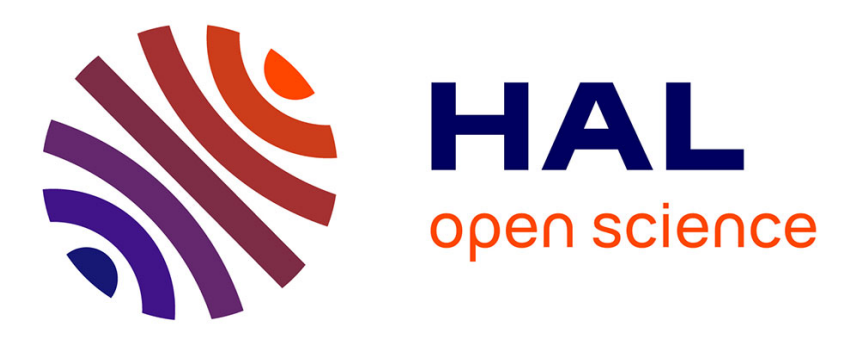

\title{
First observation of low-lying excited states in the very neutron-rich ${ }^{95} \mathrm{Kr}$
}

\author{
J. Genevey, R. Guglielmini, R. Orlandi, J.A. Pinston, A. Scherillo, G.S. \\ Simpson, I. Tsekhanovich, N. Warr
}

\section{- To cite this version:}

J. Genevey, R. Guglielmini, R. Orlandi, J.A. Pinston, A. Scherillo, et al.. First observation of low-lying excited states in the very neutron-rich ${ }^{95} \mathrm{Kr}$. Physical Review C, 2006, 73, pp.037308. 10.1103/PhysRevC.73.037308 . in2p3-00025381

\section{HAL Id: in2p3-00025381 https://hal.in2p3.fr/in2p3-00025381}

Submitted on 13 Jan 2006

HAL is a multi-disciplinary open access archive for the deposit and dissemination of scientific research documents, whether they are published or not. The documents may come from teaching and research institutions in France or abroad, or from public or private research centers.
L'archive ouverte pluridisciplinaire HAL, est destinée au dépôt et à la diffusion de documents scientifiques de niveau recherche, publiés ou non, émanant des établissements d'enseignement et de recherche français ou étrangers, des laboratoires publics ou privés. 


\title{
First observation of low-lying excited states in the very neutron-rich ${ }^{95} \mathbf{K r}$
}

\author{
J. Genevey ${ }^{1}$, R. Guglielmini ${ }^{1}$, R. Orlandi ${ }^{2,3}$, J. A. Pinston ${ }^{1}$, A. Scherillo ${ }^{2,4}$, G. Simpson ${ }^{1,2}$, \\ I. Tsekhanovich ${ }^{2}$, and N. Warr ${ }^{4}$ \\ ${ }^{1}$ Laboratoire de Physique Subatomique et de Cosmologie, \\ IN2P3-CNRS/ Université Joseph Fourier,F-38026 Grenoble Cedex, France \\ ${ }^{2}$ Institut Laue-Langevin, F-38042 Grenoble Cedex, France \\ ${ }^{3}$ Department of Physics and Astronomy, The University of Manchester, \\ Brunswick Street, M139PL, United Kingdom \\ ${ }^{4}$ Institut fur Kernphysik, Universität zu Köln \\ Zülpichestr. 77, D-50937 Köln, Germany
}

(January 12, 2006)

\begin{abstract}
Microsecond isomers of neutron-rich nuclei in the mass $A=95$ chain were investigated at the ILL reactor, Grenoble. These nuclei were produced by thermal-neutron induced fission of ${ }^{241} \mathrm{P} u$. The detection is based on time correlation between fission fragments selected by the Lohengrin mass spectrometer and the $\gamma$-rays from the isomers. In this paper, the decay scheme of an isomer of half life $1.4(2) \mu \mathrm{s}$ in ${ }^{95} \mathrm{Kr}$ is reported. It is the first time that excited states of this nucleus have been measured. These data show that this neutron rich $N=59$ isotone is spherical at low excitation energy.

PACS NUMBERS : 21.10.Tg, 23.20.Lv, 25.85.Ec, 27.60.+j
\end{abstract}

Typeset using REVTEX 
The region of the neutron-rich nuclei near $A=100$ is distinctive for its sudden change in the ground state properties of nuclei [1]. In particular, for the even ${ }_{38} \mathrm{Sr}$ and ${ }_{40} \mathrm{Zr}$ isotopes a sudden onset of strong deformation is observed at $N=60$, whereas the lighter isotopes up to $N=58$ are rather spherical. The isotones with $N=59$ neutrons are of special interest because they are just at the border of the two regions. Previous experiments have shown that their ground and low-lying states are rather spherical [2,3], while deformed bands with $\beta_{2} \sim 0.3$ are present at about $600 \mathrm{keV}$ excitation energy [4] and the maximum deformation of the region, $\beta_{2} \sim 0.44$, is reached for the $9 / 2^{+}[404]$ band recently observed at 829.8 and $1038.8 \mathrm{keV}$, in ${ }^{97} \mathrm{Sr}$ and ${ }^{99} \mathrm{Zr}$ respectively [5,6]. In the present work, we have investigated the next $N=59$ isotone, ${ }^{95} \mathrm{Kr}$. This nucleus, with a ratio $N / Z=1.69$, is located very far from the stability line and, until now, no spectroscopic information on this nucleus existed. Only a ground-state half life $\left(T_{1 / 2}=0.78 \mathrm{~s}\right)$ is reported in the literature [7].

In this work, we have searched for $\mu$ s isomers in the mass $A=95$ chain and studied their $\gamma$ decay. In the first part of the paper we describe the experimental procedure and discuss in detail the method used to identify the atomic charge $Z$ of the Kr nucleus. In the second part, we compare the level scheme of this new nucleus to the level schemes of the two other $\mathrm{Sr}$ and $\mathrm{Zr}$ isotones and deduce its nuclear structure properties.

Nuclei of mass $A=95$ chain were produced by thermal-neutron induced fission of ${ }^{241} \mathrm{Pu}$. The Lohengrin mass spectrometer was used to separate the fission fragments $(F F s)$ recoiling from a thin target of about $400 \mu \mathrm{g} / \mathrm{cm}^{2}$, according to their mass to ionic charge ratios $(A / q)$. The target was placed near the core of the reactor in a neutron flux of $5 \times 10^{14} \mathrm{n} \mathrm{cm}^{-2} \mathrm{~s}^{-1}$. At the focal plane of the spectrometer, the $F F s$ were detected in an ionization chamber filled with isobutane at a pressure of $47 \mathrm{mb}$. This ionization chamber has good nuclear charge (Z) identification. It consists of two regions of gas, $\Delta E 1=9 \mathrm{~cm}$ and $\Delta E 2=6 \mathrm{~cm}$ separated by a grid. The residual energy of the ions was measured in an array of 6 PIN diodes, which also give a rough indication of their position. This system was able to identify the nuclear charge in the $Z \sim 40$ region, with a resolution $(F W H M)$ of about two units.

The $\gamma$-rays de-exciting the isomeric states were detected by a Clover Ge detector and 
three single Ge crystals of the MINIBALL array assembled in the same cryostat. All these detectors were placed perpendicular to the ion beam. They were packed in a very close geometry, thanks to the small thickness $(6 \mathrm{~cm})$ of the ionization chamber. The total $\gamma$-ray detection efficiency was $20 \%$ and $4 \%$ for photons of $100 \mathrm{keV}$ and $1 \mathrm{MeV}$, respectively.

An event was stored on the disk of the acquisition system, each time at least one Ge detector was hit within a time range of $40 \mu$ s after the detection of a $F F$. In this case, all the signals coming from the chamber and the Ge detectors were stored, along with their time stamps.

In the present work, two $\mu$ s isomers have been observed in the $A=95$ mass chain. The strong $\gamma$ line of $260.6 \mathrm{keV}$ in Fig. 1a deexcites an isomeric level of $56.2 \mu$ s half life, already assigned to ${ }^{95} \mathrm{Y}[8]$. The two weaker $\gamma$-rays of $81.7(2)$ and $113.8(2) \mathrm{keV}$ energies and relative intensities 35(2) and 100(5), respectively, belong to a new isomer. As seen in Fig. 1b, the two transitions are in coincidence with each other. The time spectrum of the $113.8 \mathrm{keV}$ line in Fig. 2 shows that this new isomer has a half life of $1.4(2) \mu \mathrm{s}$. The large difference between the relative intensities of these two lines suggests that they have different mutipolarities. We have computed that the two transitions have comparable total intensities, $I_{T}=104(6)$ for the $81.7 \mathrm{keV}$ if one supposes that it is $E 2$ in nature, and $I_{T}=109(5)$ for $113.8 \mathrm{keV}$ if it is a $M 1$ or $106(5)$ if it is an $E 1$. This result is obtained assuming pure mutipolarities in the calculations.

The method used for the nuclear charge $(Z)$ identification of this new isomer of mass 95 , is shown in Fig. 3. Coincidence measurements were performed between the incoming ions and the $\gamma$ rays deexciting the isomers, and the $\Delta E 1$ energy lost in the first region of gas was measured. To improve the $Z$ resolution of the chamber, only a part of the whole data set was used in the analysis. More precisely, only the ions in coincidence with one of the six PIN diodes were analysed. The $Z$ calibration of the mass 95 isobars is deduced from the energy lost in the first stage gas of the ionization chamber $(\Delta E 1)$ by the ions of ${ }^{95} \mathrm{Y}$ and ${ }^{95} \mathrm{Sr}$ respectively. The ${ }^{95} \mathrm{Y}$ isomer was already known and its $\Delta E 1$ is shown in Fig. 3a. The $\Delta E 1$ spectrum for the whole mass chain 95 is shown in Fig. 3b. The values of the independent 
yields of ${ }_{36} \mathrm{Kr},{ }_{36} \mathrm{Rb},{ }_{36} \mathrm{Sr},{ }_{36} \mathrm{Y}$ isobars, produced by thermal-neutron induced fission of ${ }^{241} \mathrm{Pu}$, are $7.3 \times 10^{-5}, 4.9 \times 10^{-3}, 2.7 \times 10^{-2}$, and $7.8 \times 10^{-3}$, respectively [9]. Consequently, the yield of ${ }^{95} \mathrm{Sr}$ is much stronger than the yields of the other isobars and corresponds to the centroid of the $(\Delta E 1)$ distribution. The asymmetry observed in this $\Delta E 1$ spectrum reflects the difference between the relative yields of ${ }^{95} \mathrm{Rb}$ and ${ }^{95} \mathrm{Y}$. Knowing the centroids of the $\Delta E 1$ spectrum for ${ }^{95} \mathrm{Y}$ and ${ }^{95} \mathrm{Sr}$, it is possible to extrapolate the centroid position of the ${ }^{95} \mathrm{Rb}$ and ${ }^{95} \mathrm{Kr}$ distributions. A precise knowledge of the energy lost in the gas for the different isotopes is not necessary, and only channel numbers are plotted on the x horizontal-axis of Fig. 3. The $\Delta E 1$ spectrum of the new isomer is shown in Fig. 3c. It was obtained by coincidences between the incoming ions and the $\gamma$-rays of the isomer. Moreover, to reduce the influence of random events on the $\Delta E 1$ spectrum, the last $4 \mu$ s from the $40 \mu$ s time window spectrum was subtracted from the first $4 \mu$ s spectrum gated with the isomeric $\gamma$-rays. This is because the last $4 \mu \mathrm{s}$ countains only random background coincidences. The large fluctuations around zero observed in the right part of the figure are caused by the subtraction. The position of the centroid of the $\Delta E 1$ distribution allows the nuclear charge $Z=38$ to be assigned to this new isomer unambiguously.

We have deduced from the $\gamma$-ray spectra the ratio of the fission yields for the two isomers of ${ }^{95} \mathrm{Kr}$ and ${ }^{95} \mathrm{Y}$. The measured value of Yield $(\mathrm{Kr}) /$ Yield $(\mathrm{Y})=9.3 \times 10^{-3}$ is of the same order of magnitude as the ratio of the yields, $2.1 \times 10^{-2}$, measured for the ground states. This result is an indirect proof of the assignment of the isomer to the Kr nucleus.

In Fig. 4, the level scheme of the new isomer found in ${ }^{95} \mathrm{Kr}$ is shown and compared with the previously known isomers in the isotones of ${ }^{97} \mathrm{Sr}$ and ${ }^{99} \mathrm{Zr}[2,3,5,6]$. During this run, we have also remeasured the half-life of the $829.8 \mathrm{keV}$ isomer in ${ }^{97} \mathrm{Sr}$, for which very different values are reported in the literature $[6,10,11]$. A very precise value, $T_{1 / 2}=526(13) n s$, was found and is reported in the level scheme of ${ }^{97} \mathrm{Sr}$. This point is discussed in a forthcoming publication [12]. In these three nuclei, the low-lying isomer decays by an E2 transition. The measured $B(E 2)$ values, $1.33(5), 1.75(10)$ and $1.47(27) W u .$, for ${ }^{99} \mathrm{Zr},{ }^{97} \mathrm{Sr}$ and ${ }^{95} \mathrm{Kr}$, respectively, are comparable, which suggests that the three transitions have an analogous 
nature and that the three isotones have the same spins. Using this hypothesis, the 113.8 $\mathrm{keV}$ transition then takes place between the $3 / 2^{+}$and $1 / 2^{+}$levels and is expected to be $M 1$ in nature. This interpretation agrees with the rather pure $M 1$ or $E 1$ multipolarity measured for this transition and agrees with the pure $M 1$ transitions previously measured in ${ }^{97} \mathrm{Sr}$ and ${ }^{99} \mathrm{Zr}$. These common behaviours between ${ }^{95} \mathrm{Kr}$ and the two other isotones suggest that they have comparable structure at low energy. Consequently, the ground and the two first excited states of these three isotones are very likely spherical, as suggested by the measured $B(E 2)$ values, and their dominant configurations are the $\nu s_{1 / 2}, \nu d_{3 / 2}$ and $\nu g_{7 / 2}$ shell-model states, respectively. One may notice that their energies change very little between ${ }^{99} \mathrm{Zr}$, which is quite close to the line of stability and ${ }^{95} \mathrm{Kr}$, which is very far from it.

In contrast, we have not observed the $9 / 2^{+}[404]$ strongly deformed isomer, present in the two other isotones [5]. The non observation of this isomer means that either this level does not exist in ${ }^{95} \mathrm{Kr}$, it is too weakly fed by fission, or its half life is shorter than about $0.5 \mu \mathrm{s}$, because the flight time throw the Lohengrin spectrometer is about $1.7 \mu \mathrm{s}$. 


\section{FIGURES}

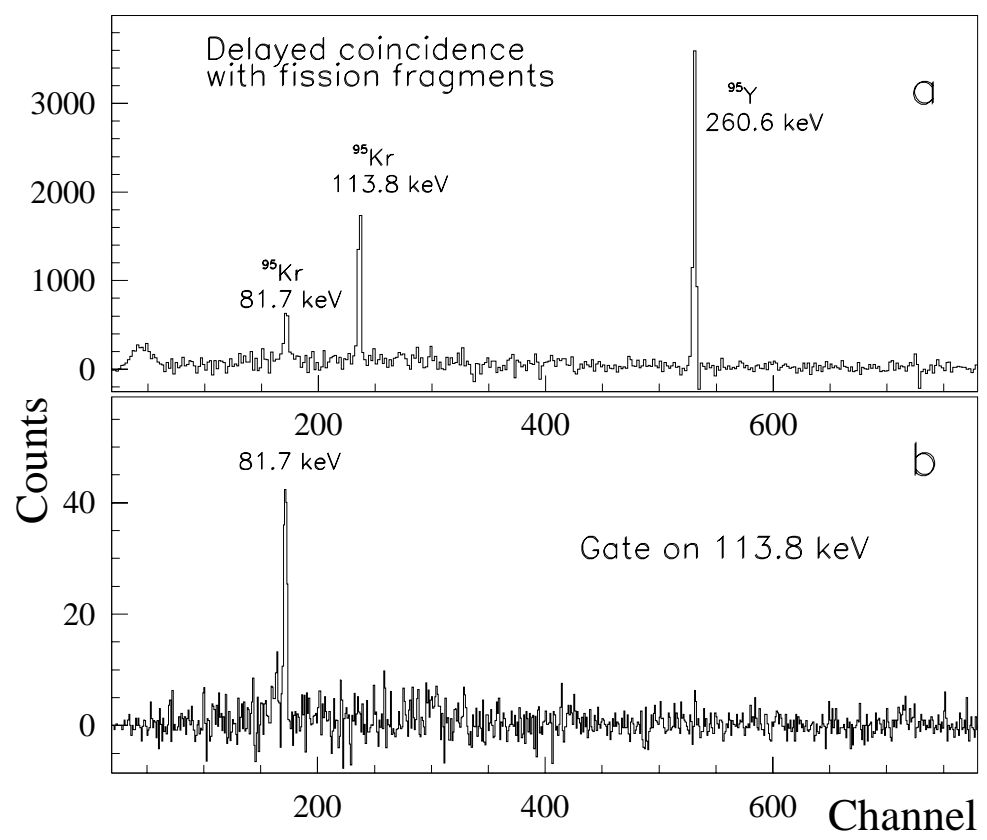

FIG. 1. (a) $\gamma$ decay spectrum of the ${ }^{95} \mathrm{Kr}$ and ${ }^{95} \mathrm{Y}$ isomers. (b) Coincidence spectrum gated on the $113.8 \mathrm{keV} \gamma$ ray.

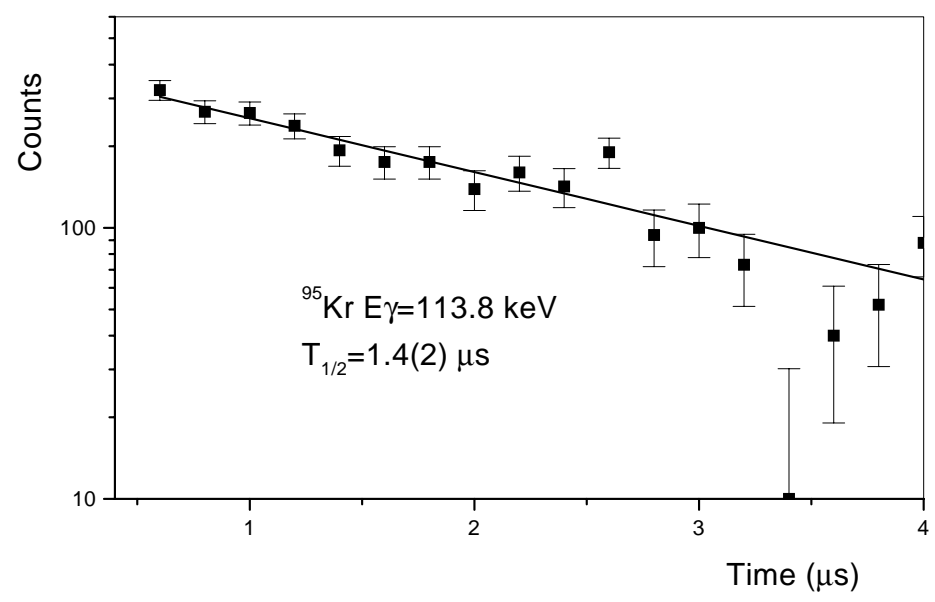

FIG. 2. Time spectrum of the $113.8 \mathrm{keV} \gamma$ ray. 


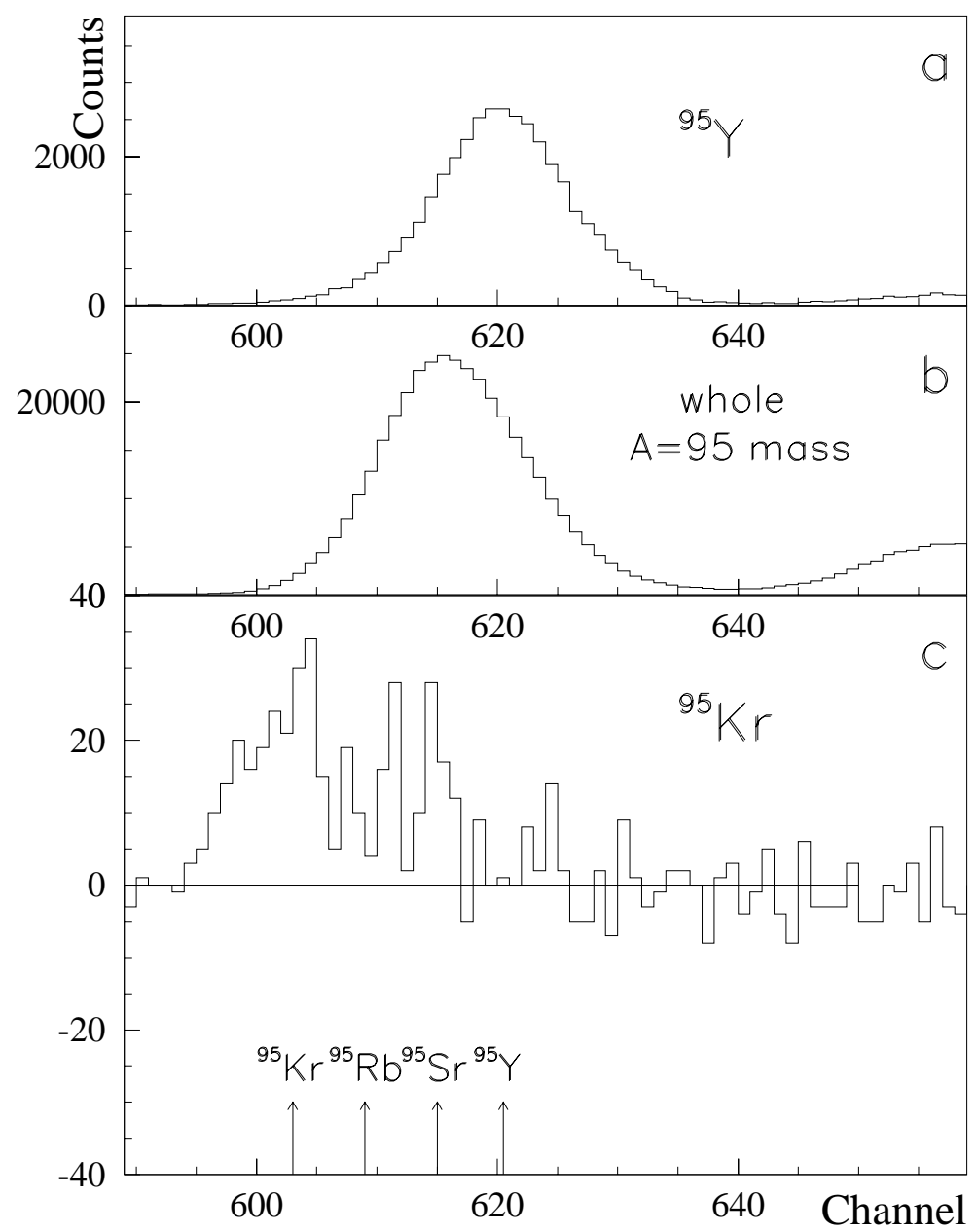

FIG. 3. Energy lost in the first step of gas $\Delta E 1$, for (a) the ${ }^{95} \mathrm{Y}$ isomer, (b) the whole $A=95$ mass chain, (c) the ${ }^{95} \mathrm{Kr}$ isomer. 


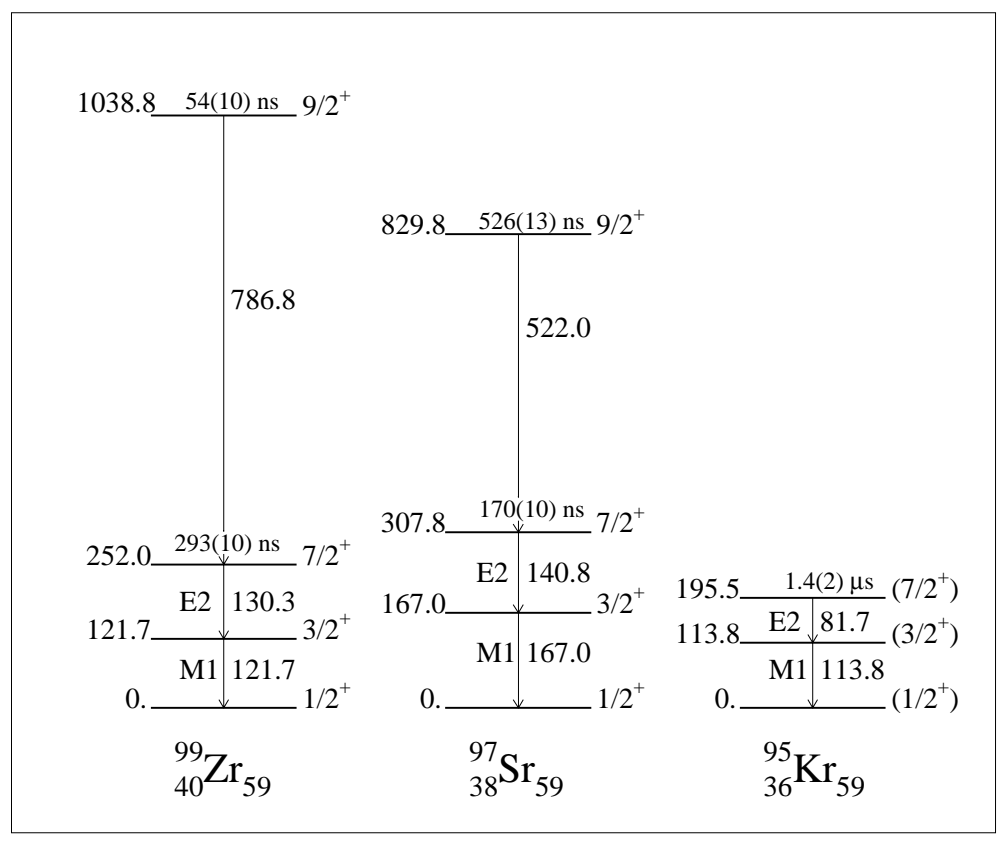

FIG. 4. Decay schemes of the ${ }^{99} \mathrm{Zr},{ }^{97} \mathrm{Sr}$ and ${ }^{95} \mathrm{Kr}$ isomers.

\section{ACKNOWLEDGMENTS}

Two of the authors (A. S. and N. W.) acknowledge support from BMBF under grant O6K-167. We would like to thank the MINIBALL collaboration for the use of one of their detectors. 


\section{REFERENCES}

[1] J. L. Wood, K. H. Heyde, N. Nazarewicz, M. Huyse and P. Van Duppen, Phys. Rep. 215, 101 (1992).

[2] K. L. Kratz et al., Z. Phys. A, 312, 43 (1983).

[3] L. K. Peker, Nucl. Data Sheets 73, 1 (1994).

[4] W Urban et al., Nucl. Phys. A689, 605 (2001).

[5] W. Urban et al., Eur. Phys. J. A22, 241 (2004).

[6] J. K. Hwang et al., Phis. Rev. C 67, 054304 (2003).

[7] H. Ahrens, P. Patzelt and G. Herrmann, J. Inorg. Nucl. Chem. 38, 191 (1976).

[8] B. Pfeiffer, E. Monnand, J. A. Pinston, F. Schussler, G. Jung, J. Munzel and H. Wolnik, Proc. Int. Conf. Far from Stability, Helsingor, Denmark 7-13 June 1981, CERN report 81-09, p. 423.

[9] JEF2, Joint European File, a computed file of fission data maintained by NEA.

[10] E. Monnand,J. A. Pinston, F. Schussler, J. B. Battistuzzi, K. Kamada, H. Lawin, K. Sitemich and B. Pfeiffer, report CEA-N-2176, p.20 (1980).

[11] B. Pfeiffer and J.A. Pinston, www.nndc.bnl.gov/useroutput/AR-88270-1.

[12] A. Zlomamaniec, H. Faust, J. Genevey, J. A. Pinston, T. Rząca-Urban, G. S. Simpson, I. Tsekhanovich and W. Urban, accepted for publication in Phys. Rev. C journal. 